\title{
Mapping SERS in CB:Au Plasmonic Nano-Aggregates
}

Cloudy Carnegie, Rohit Chikkaraddy, Felix Benz, Bart de Nijs, William Deacon, Matthew Horton, Wenting Wang, Charlie Readman, Steven J. Barrow, Oren A Scherman, and Jeremy J. Baumberg ACS Photonics, Just Accepted Manuscript • DOI: 10.1021/acsphotonics.7b00902 • Publication Date (Web): 16 Oct 2017 Downloaded from http://pubs.acs.org on October 18, 2017

\section{Just Accepted}

"Just Accepted" manuscripts have been peer-reviewed and accepted for publication. They are posted online prior to technical editing, formatting for publication and author proofing. The American Chemical Society provides "Just Accepted" as a free service to the research community to expedite the dissemination of scientific material as soon as possible after acceptance. "Just Accepted" manuscripts appear in full in PDF format accompanied by an HTML abstract. "Just Accepted" manuscripts have been fully peer reviewed, but should not be considered the official version of record. They are accessible to all readers and citable by the Digital Object Identifier (DOI®). "Just Accepted" is an optional service offered to authors. Therefore, the "Just Accepted" Web site may not include all articles that will be published in the journal. After a manuscript is technically edited and formatted, it will be removed from the "Just Accepted" Web site and published as an ASAP article. Note that technical editing may introduce minor changes to the manuscript text and/or graphics which could affect content, and all legal disclaimers and ethical guidelines that apply to the journal pertain. ACS cannot be held responsible for errors or consequences arising from the use of information contained in these "Just Accepted" manuscripts. 


\title{
Mapping SERS in CB:Au Plasmonic Nano-Aggregates
}

\author{
Cloudy Carnegie ${ }^{1}$, Rohit Chikkaraddy ${ }^{1}$, Felix Benz ${ }^{1}$, Bart de Nijs ${ }^{1}$, William Deacon ${ }^{1}$, Matthew \\ Horton $^{1}$, Wenting Wang ${ }^{1,2}$, Charlie Readman ${ }^{1,2}$, Steven J. Barrow ${ }^{2}$, Oren A Scherman ${ }^{2}$, \\ Jeremy J. Baumberg ${ }^{*}, 1$ \\ ${ }^{1}$ NanoPhotonics Centre, Cavendish Laboratory, University of Cambridge, Cambridge, CB3 OHE, UK \\ ${ }^{2}$ Department of Chemistry, University of Cambridge, Lensfield Road, Cambridge CB2 1EW, UK
}

KEYWORDS: plasmon, sensing, aggregate, nanogap, SERS, nano self-assembly

\begin{abstract}
In order to optimize surface-enhanced Raman scattering (SERS) of noble metal nanostructures for enabling chemical identification of analyte molecules, careful design of nanoparticle structures must be considered. We spatially map the local SERS enhancements across individual microaggregates comprised of monodisperse nanoparticles separated by rigid monodisperse $0.9 \mathrm{~nm}$ gaps and show the influence of depositing these onto different underlying substrates. Experiments and simulations show that the gaps between neighbouring nanoparticles dominate the SERS enhancement far more than the gaps between nanoparticles and substrate.

Metal nanoparticle aggregates support a large number of plasmonic hotspots within the inter-nanoparticle gaps, which can be used to probe molecular vibrations of analytes through surface-enhanced Raman scattering (SERS) ${ }^{1,2}$. The high sensitivity of SERS and its consequent utilisation as a sensing technique has been demonstrated in a number of studies ${ }^{3-11}$. Various methods have been used to form aggregates from colloidal nanoparticles, including DNA $\mathrm{A}^{7,12}$, proteins $\mathrm{s}^{9,13}$, dyes ${ }^{14}$, alcohols ${ }^{6}$, polymers ${ }^{15,16}$, and optical fields ${ }^{17-20}$, however these methods generally form agglomerates with wide variation in configurations and gap sizes. In such systems a large number of molecules across multiple hot-spots are probed in time, producing only a collective overall SERS signal. For typical colloidal aggregation onto a substrate, the very wide range in gap sizes produces broad absorption across the visible and near-infrared spectrum, and sporadically located 'hot-spots' in real space ${ }^{21,22}$. In all these hot-spot crevices, each molecule sees a different orientation, strength, and resonant frequency of the optical-excited plasmon field. This means that the SERS averages over all field-molecule configurations, which is undesirable for studying selection rules, nonlinear vibrational phenomena, and for robust uniform sensing.
\end{abstract}

Here, we assemble and probe nanoparticle aggregates with precisely fixed sub-nm gaps, and precisely oriented molecules and optical fields ${ }^{23}$. In order to map the Raman signal from individual aggregates without having to track these as they diffuse in solution, we bind them onto solid substrates. The optimal SERS emission is found to be on $\mathrm{Au}$ substrates rather than $\mathrm{SiO}_{2}$ or $\mathrm{Si}$, and can be explained from image charge interactions. Placing analyte molecules into these precise sub-nm hotspots of high plasmonic enhancement allows us to spatially map the signals across single aggregates. The tight focusing of light possible in this geometry gives better collection and thus higher SERS signals than with lower NA objectives

*jjb12@cam.ac.uk 
averaging over many aggregates at once, either on substrates or in solution. This enables the robust prospect of sensing sub-nanomolar concentrations, for use in gas sensing and flow microfluidics for pharmaceuticals and biological cell sensing.

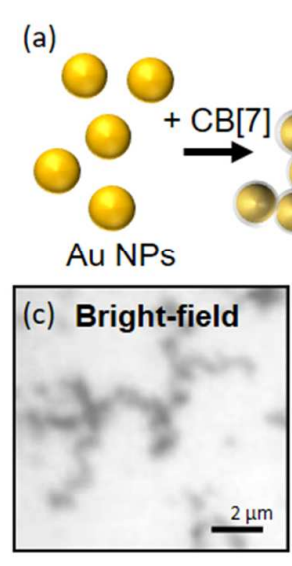

(e) Extinction

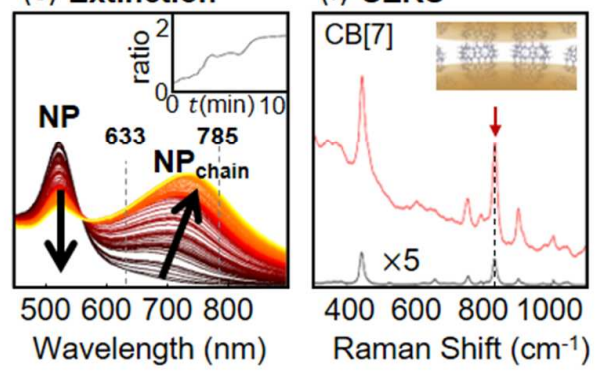

Figure 1. (a,b) Nanoparticle aggregation by addition of cucurbit[7]uril (CB[7]), shown in (b). (c) Bright-field image of aggregate dried onto gold substrate. (d) Schematic distribution of chain (red) and dimer (blue) plasmon modes. (e) Time-resolved extinction spectra over 10 mins, showing decrease in single-particle mode and increase in chain modes (laser wavelengths shown dashed, inset shows ratio of coupled mode to transverse mode over time. (f) SERS spectrum of Au:CB[7] aggregate in solution (red) and CB[7] powder for reference (black, $\mathrm{x} 5$ for visibility). Red arrow indicates CB vibration mapped in later images.

The metal nanoparticle aggregates are self-assembled in solution by the addition of the spacer molecule cucurbit[7]uril to $40 \mathrm{~nm}$ diameter colloidal gold nanoparticles. Cucubit[n]urils ( $\mathrm{CB}[n] \mathrm{s})$ are a series of rigid and initially-empty barrel-shaped molecules with hydrophobic cavities and hydrophilic carbonyl portals which bind strongly to gold (Fig.1a,b) ${ }^{24}$. Previous work shows that $\mathrm{CB}[n]$ readily aggregates Au nanoparticles forming a precise interparticle separation of $0.9 \mathrm{~nm}$, and providing a reliable way to form optically-active long aggregate chains with fractal-like structures ${ }^{25}$. Such nanoscale gaps between plasmonic metals trap incident light at specific resonant wavelengths, greatly enhancing the optical field and thus the Raman scattering of molecules within the gap. Due to their stiff hollow structures, $\mathrm{CB}[n] \mathrm{s}$ can encapsulate hydrophobic or cationic molecules, making this construct suitable for gas and chemical sensing. Other macromolecules such as cyclodextrin have also been shown to encapsulate analyte molecules for sensing with SERS ${ }^{26,27}$, however the inter-nanoparticle gaps formed are not as rigidly spaced as those formed with $\mathrm{CB}[n]$, meaning the plasmon cannot couple to form chain plasmon modes in the same way. Additionally $\mathrm{CB}[7]$ is a member of a family of $\mathrm{CB}[n]$ molecules, which can be selectively chosen to suit the particle analyte of interest. Normally such aggregates are observed in solution, where they fill only a small amount of the optical focal region, thus reducing the available SERS signal. Here, once the aggregates are formed, they are drop cast onto different substrates at sufficiently low concentration to image them individually 
(although binding them in-situ within microfluidic channels is also effective). The aggregates are visible in bright-field images (Fig.1C), with corresponding scanning electron micrographs showing their 3D fractal geometry (SI). The strong CB-Au binding ( $\sim 0.3 \mathrm{eV} /$ molecule) means that drop casting aggregates onto a substrate does not markedly change their 3D structure ${ }^{28}$.

Because the identical plasmonic gaps in such assemblies support degenerate localised plasmon modes, charge oscillations in one nano-gap mix strongly with charge oscillations of surrounding nano-gaps, resulting in spatially-distributed plasmonic modes. These complex modes can be broken down into 3 main types, which are observed in real-time optical scattering as the nanoparticles aggregate together (Fig. 1d,e). Further explanation of this aggregation has been extensively studied in our previous work ${ }^{25}$. Initially the individual nanoparticles support a monomer plasmon mode at $530 \mathrm{~nm}$. Adding CB[7] first induces dimerization resulting in a new mode at $640 \mathrm{~nm}$ while the single particle mode decreases. At later times, higher wavelength chain modes emerge as the aggregate size increases, red-shifting with increasing chain length. Although the single-particle and dimer modes are still supported at the peripheries of large aggregates, the optical response is dominated by chain modes which are delocalised across the entire structure. Nanoparticle aggregates saturate in size once surface coverage of CB[7] limits the probability of further aggregation.

The enhanced optical field within each gap allows the SERS of the surface-bound CB in the gaps to be observed, giving a number of characteristic peaks (Fig. 1f). Here, we use the Raman strength of one particular CB vibration (the $832 \mathrm{~cm}^{-1}$ peak corresponding to a ring-breathing mode) as a marker of the SERS activity at each gap site positioned precisely in between nanoparticles. By spatially mapping the intensity of this $\mathrm{CB}$ [7] peak, the optical field variations can be tracked over the aggregate structure. As well as the peak intensity of this vibrational mode, the plasmonic background can also be mapped across the aggregate area, and its intensity is found to be directly correlated to that of the SERS peaks.

To understand how to optimise the SERS emission from single aggregates, different substrates are compared (Fig.2). Besides the plasmonic coupling between NPs, there can also be coupling to the underlying substrate $e^{29,30}$ and this will be different for the 3 types of plasmonic mode within such aggregates $^{29}$ (see below). After drop-casting onto the three substrate types (template-stripped gold, bare silicon wafer, and glass coverslip), SERS spectra are mapped using a confocal pinhole with either a $633 \mathrm{~nm}$ or $785 \mathrm{~nm}$ laser, across $12 \mu \mathrm{m} \times 12 \mu \mathrm{m}$ areas in $100 \mathrm{~nm}$ steps (Fig. 2a-c). The integrated area under the $832 \mathrm{~cm}^{-1}$ ring-breathing mode is extracted for each spatial position to give a map of SERS emission, normalised to the incident laser power. The aggregate shape is clearly reproduced in these SERS maps on all the substrates, although the signal is much reduced on $\mathrm{Si}$. The strong correlation between bright-field scatter and SERS signals across each point in the image maps is evident for all 3 systems (Fig.2d). 

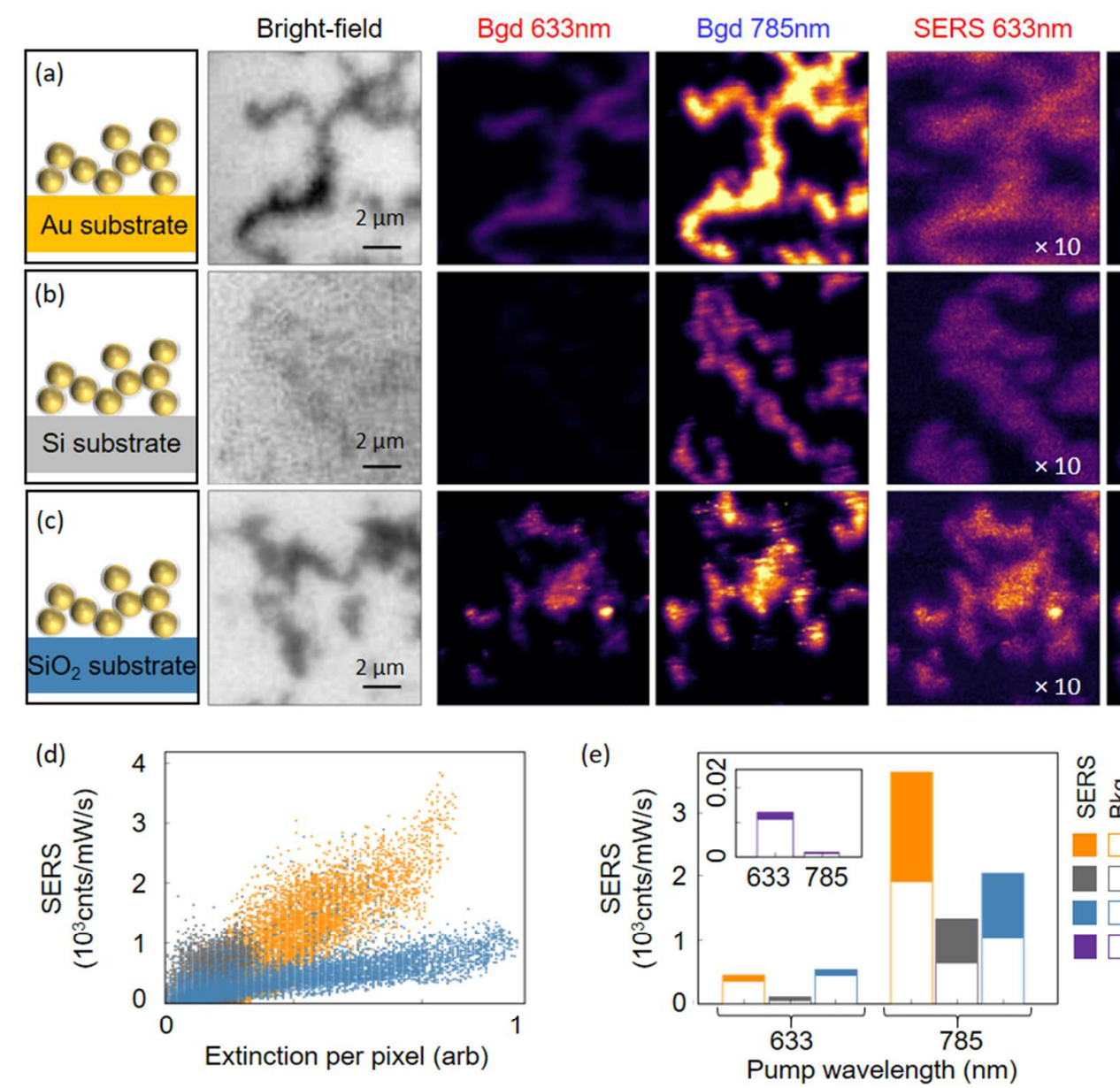

(b)

(1)

4

(e)

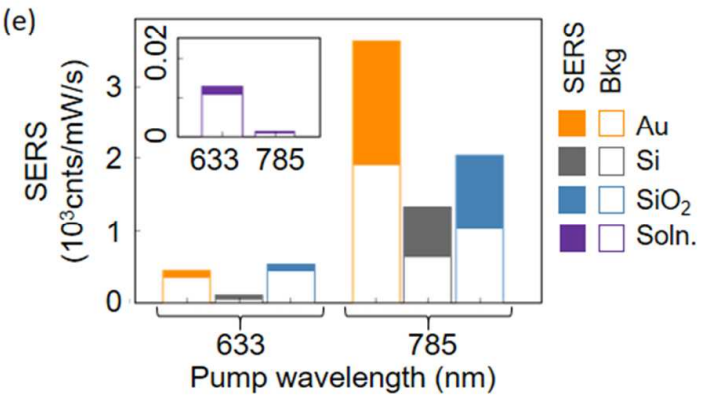

Figure 2. (a-c) Bright-field images and intensity maps showing SERS signals and plasmonic background for AuNP:CB aggregates drop-cast onto (a) gold, (b) silicon, and (c) silicon dioxide substrates. Identical colour scales except where indicated. (d) Scatter plot showing correlation between BF extinction of each pixel and its SERS intensity with $785 \mathrm{~nm}$ excitation laser, for all three substrates. (e) Stacked bar-chart showing averaged SERS (filled) and background (open) across the mapped aggregate image for different substrates, at excitation wavelengths of 633 and $785 \mathrm{~nm}$. Inset shows equivalent measurement for AuNPs:CB in solution, with pump laser $633 \mathrm{~nm}$ (left) and $785 \mathrm{~nm}$ (right).

Since on average, scattering is related to the number of CB:Au nanogaps at each pixel, this shows that SERS linearly adds from each nanogap ${ }^{31}$. This is analogous to the typical linear increase of both SERS and extinction with the number of nanoparticles when no coupling between them is present. In order to quantify the efficiency of each aggregate system as a SERS substrate, the average SERS intensity for the aggregate (within its interior region on the map) was calculated. This is shown by the stacked bars in Fig. $2 \mathrm{e}$, with the open bar components indicating the strength of the background signals. The $785 \mathrm{~nm}$ laser gives consistently higher signals than using a $633 \mathrm{~nm}$ laser, due to its resonance with the chain modes of the Au aggregates, (Fig. 1e, dashed). It also gives SERS peaks of similar strength to the SERS background, while the background dominates at $633 \mathrm{~nm}$. Comparing the SERS intensity between aggregates deposited on the different substrates, shows that deposition onto a gold substrate gives the strongest signals. As we demonstrate below, this is due to the plasmonic coupling of gap plasmons to image charges that occurs when metal nanostructures are placed above gold mirrors. However the SERS signal is only two-fold weaker for aggregates deposited on $\mathrm{SiO}_{2}$, where there is no such plasmonic coupling to the substrate, suggesting 
this coupling is not the dominant factor for SERS enhancement. By calibrating the bright-field reflectance, we are able to estimate that each pixel contributes signal from 1 NP-NP junction containing $\sim 100$ closepacked CB molecules, and gives a total SERS integrated signal of $1000 \mathrm{cts} / \mathrm{mW} / \mathrm{s}$. Thus we estimate that each CB molecule contributes $10 \mathrm{cts} / \mathrm{mW} / \mathrm{s}$, in line with the expected non-resonant SERS cross-section and the plasmon local field, matching SERS observations from single nanoparticle gaps containing CBs in the nanoparticle-on-mirror configuration ${ }^{30,32}$. With unity CCD gain and the optimised system throughput for detection of $\sim 10 \%$ this suggests 100 SERS photons emitted $/ \mathrm{mW} / \mathrm{s} / \mathrm{molecule}$ for the strongest lines in these non-aromatic molecules.

To compare with the colloidally-suspended aggregates normally used, equivalent measurements of $C B[7]$ SERS signals are taken with Au:CB aggregates in solution (inset Fig. 2e). The SERS intensity is found to be 1000-fold smaller when compared to aggregates deposited on $\mathrm{SiO}_{2}$, which is the reduction expected due to the dilution of aggregate density within the focal optical volume probed at any time, averaged by diffusion. The amplification in signal due to deposition on a substrate is greater when using a gold substrate, due to the extra degree of plasmonic enhancement. Conversely it is lessened for Si due to absorption into the substrate. Surprisingly we find that the signal from the $785 \mathrm{~nm}$ laser in such solution aggregates is now 5fold weaker than from $633 \mathrm{~nm}$, the opposite ratio to the dried aggregates, even though wet and dry extinction spectra can be similar as we now investigate.

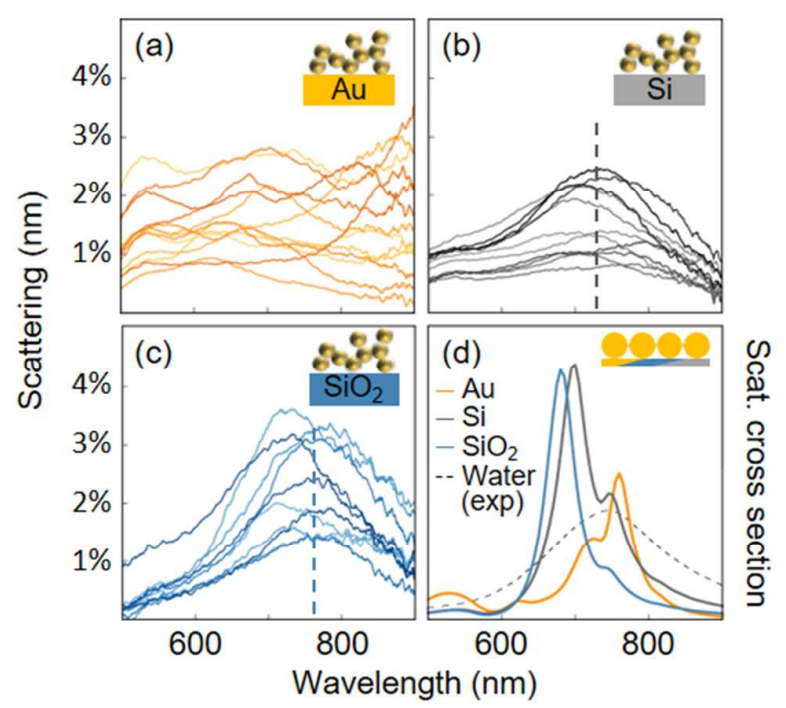

Figure 3. (a-c) Dark-field scattering spectra of aggregates on (a) $\mathrm{Au},(\mathrm{b}) \mathrm{Si}$, and (c) $\mathrm{SiO}_{2}$. (d) FDTD simulated scattering cross sections of a 4 nanoparticle chain spaced above $\mathrm{Au}, \mathrm{Si}$, and $\mathrm{SiO}_{2}$ substrates. Dashed line shows experimental results for scattering of the aggregates in water with no substrate.

To understand this more clearly, dark-field scattering measurements are taken to probe the plasmon resonances of a number of aggregates on the different substrates (Fig. 3a-c). These experimental scattering spectra are taken over an assortment of representative aggregates and positions, evidencing expected spatial inhomogeneities (Fig.1d) but generally common features on each substrate. While a weak singleparticle mode at $530 \mathrm{~nm}$ is always visible, the dominant chain modes are clearly seen to longer wavelengths. These red-shifted peak positions depend on the coupling strength, set by how effectively nanoparticle charge oscillations generate image charges in each substrate. When on $\mathrm{SiO}_{2}$ the coupled mode is similar as in solution, however it shifts to shorter wavelengths and weakens when placed on Si due to the 
damping by absorption in the semiconductor. This is also the reason that SERS is so much weaker on the Si substrates, since both elastic and inelastic scattering are similarly affected.

By contrast, aggregates on gold instead show especially large variation in this coupled-mode spectral position, shifted far more into the infrared and with multiple resonances. This higher degree of coupling to the gold substrate can be confirmed using full 3D finite-difference time domain (FDTD) simulations. We mimic the experimental configuration with a toy model comprised of a chain of 4 nanoparticles spaced above substrates of gold, $\mathrm{Si}$ or $\mathrm{SiO}_{2}$ (Fig. 3d). ${ }^{33,34}$ The spacer layers at each NP-NP junction as well as the substrate-NP junction have refractive index of 1.4 to match that of $\mathrm{CB}[7]^{30}$. The scattering cross section as a function of wavelength is obtained for normal plane-wave illumination with polarization along the chain. Due to computational intractability it is not feasible to fully model entire aggregates with complex geometries $^{22}$, hence the modal positions calculated are only indicative (and blue-shifted from experiment as expected for shorter chains ${ }^{35}$ ). The stronger coupling of Au substrates to the CB-spaced plasmonic gaps indeed gives higher wavelength modes in the FDTD simulated spectra, as compared to the non-metallic substrates.

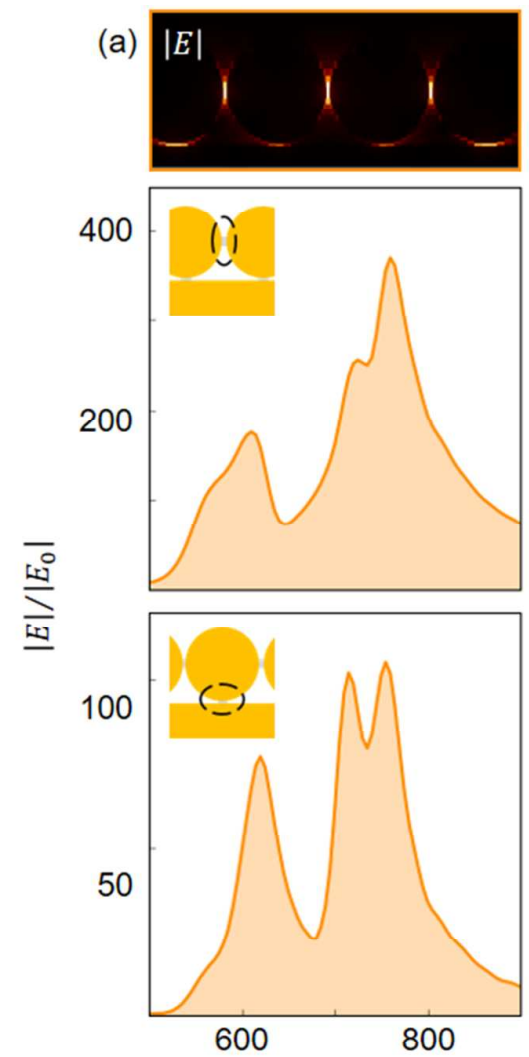

(b)

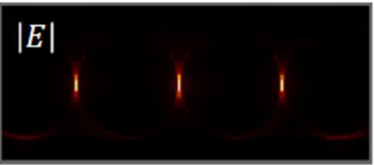

(c)
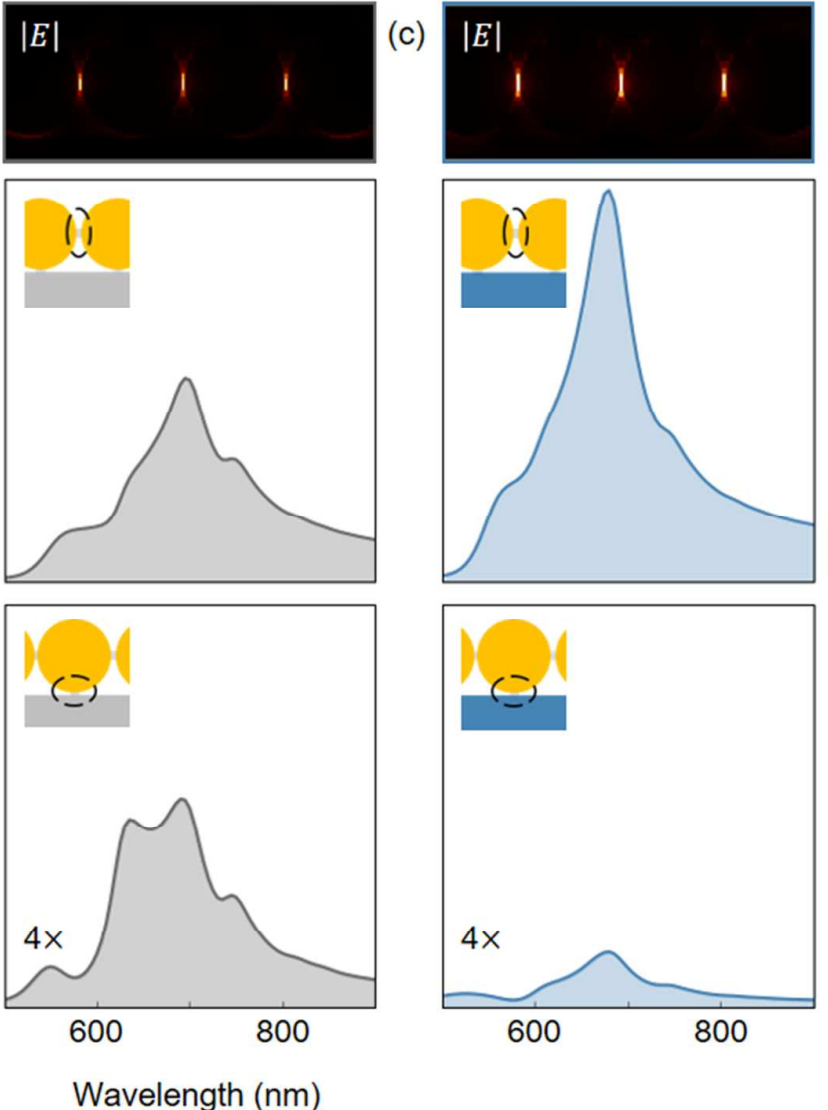

Figure 4. (a-c) FDTD simulations showing |E| surrounding a 4-nanoparticle chain spaced $0.9 \mathrm{~nm}$ above an infinite substrate of Au, $\mathrm{Si}, \mathrm{SiO}_{2}$ respectively. Each is shown for the relevant resonant wavelength and colour scales are identical. Graphs below show the near-field spectra at the central NP-NP and substrate-NP junction for each system (dashed region marked).

To identify which regions within the aggregates are involved in the substrate coupling, near-field enhancements, $|E|$ are extracted from the simulations (Fig.4). We distinguish hotspots at the junction 
between two nanoparticles (NP-NP junctions) and those at the interface between substrate and nanoparticle (sub-NP junctions). At the substrate-NP junction, the near-field spectra show much higher gap field intensities with the gold substrate. Indeed, this enhancement is often used for single NPs on Au substrates (known as nanoparticle-on-mirror ${ }^{29,30}$ ). By contrast in the NP-NP junctions, aggregates on $\mathrm{SiO}_{2}$ or $\mathrm{Au}$ have comparable near-field intensities, with $\mathrm{Si}$ again weaker from absorption. Additionally, field intensities in NP-NP junctions are many-fold stronger than in the substrate-NP junction in all cases (Fig. 4 lower graphs). This suggests that for dried aggregate geometries the field between nanoparticles is largely responsible for hotspot effects such as SERS, confirming what was indicated by the intensities of SERS maps in Fig.2e. On the other hand, the near-field spectra of the NP-NP junctions are red-shifted for the Au substrate (compared to on the $\mathrm{SiO}_{2}$ substrate), showing that they are well-coupled to the substrate-NP junctions. For this reason careful choice of substrate is needed in order to maximise SERS signal enhancements.

We thus evidence how signals can be enhanced 1000-fold by casting Au:CB SERS aggregates onto glass, with Au substrates giving an extra factor of two gain due to image charges. Excitation with $785 \mathrm{~nm}$ laser light improves the signal-to-noise in SERS emission, due to the decreased background contributions (which come from light emission by electronic Raman scattering coupled by the plasmons ${ }^{36,37}$ ). The linearity of SERS emission with number of junctions implies each sums incoherently into the total. Our signals of $>10^{3}$ counts $/ \mathrm{mW} / \mathrm{s}$ (or $>10$ counts $/ \mathrm{mW} / \mathrm{s}$ per molecule, input power density $650 \mu \mathrm{W} / \mu \mathrm{m}^{2}$ ) from electronically non-resonant, non-aromatic molecules provides strong encouragement for sensing applications, for instance of neuro-active molecules in urine ${ }^{38}$. These aggregates remain stable for SERS for indefinite periods, and thus form stable SERS substrates in microfluidics contexts.

In conclusion, we present detailed analysis of the factors controlling the SERS intensity from nanoparticle aggregates with precise identical $0.9 \mathrm{~nm}$ gaps, using the $\mathrm{CB}$ spacer as a vibrational marker. Our results show that excitation with $785 \mathrm{~nm}$ laser light gives the strongest Raman signals from analytes, due to optimal overlap with the resonance wavelength of the chain modes within the aggregates. We additionally demonstrate how deposition of aggregates onto a substrate enhances the SERS signal by a factor of 1000, and even more if plasmonically active substrates are used. We illustrate that the inter-nanoparticle gaps dominate the near-field Raman response rather than the coupling of aggregate to image charges within the substrate. Our findings indicate the need for careful consideration of nano-systems when using surfaceenhanced Raman scattering as a sensing technique, and pave the way for robust sensitive measurements suited to personalised healthcare technologies.

\section{Methods}

Sample preparation: Silicon wafers are purchased from Si-Mat, and $\mathrm{SiO}_{2}$ cover slips ( $50 \mu \mathrm{m}$ thickness) from Thermo Scientific. Atomically smooth gold substrates are prepared by e-beam evaporation of $100 \mathrm{~nm}$ gold layers onto a clean silicon wafer. This is then heated to $60^{\circ} \mathrm{C}$, and small pieces of silicon glued to it and then cured, before being peeled off to reveal a ultra-smooth gold surface. The nanoparticle aggregates were self-assembled by adding $25 \mu \mathrm{l}$ of $1 \mathrm{mM} \mathrm{CB}$ [7] solution to $1000 \mu \mathrm{l} 40 \mathrm{~nm}$ gold nanoparticles in a citrate buffer (BBI solutions). The aggregates were then drop cast onto the 3 substrates, left to dry and then rinsed with deionized water.

Simulation: Finite-difference time-domain simulations were carried out using Lumerical FDTD Solutions v8.12. A simple aggregate geometry was modelled as 4 gold spheres spaced above an infinite sheet of Au, 
$\mathrm{Si}$, or $\mathrm{SiO}_{2}$ by 4 dielectric cylinders of height $0.9 \mathrm{~nm}$ and refractive index 1.4, to correspond to the $\mathrm{CB}[7]$ layer. The 4 spheres are also spaced apart from each other by identical cylinders. The construct was illuminated by a normal plane wave.

Raman measurements: Raman microscopy was performed using a Renishaw inVia Raman microscope, using a 100X objective with numerical aperture $N A=0.75$. Excitation was performed with either $633 \mathrm{~nm}$ or $785 \mathrm{~nm}$, with a power density of $650 \mu \mathrm{W} / \mu^{2}$ for each. All measurements were normalised to laser power and integration time. Measurements were taken with unity CCD gain.

\section{Supporting Information}

Scanning electron micrographs show 3D fractal geometry of $80 \mathrm{~nm}$ nanoparticle aggregates self-assembled via addition of cucurbit[7]uril.

\section{Acknowledgements}

We acknowledge financial support from EPSRC Grants EP/ G060649/1, EP/K028510/1, and EP/L027151/1 and ERC Grant LINASS 320503. C.C acknowledges support from the UK National Physical Laboratories. R.C. acknowledges support from the Dr. Manmohan Singh scholarship from St. John's College. F.B. acknowledges support from the Winton Programme for the Physics of Sustainability. S.J.B. acknowledges support from the European Commission for a Marie Curie Fellowship (NANOSPHERE, 658360)

\section{References}

(1) Stiles, P. L.; Dieringer, J. A.; Shah, N. C.; Van Duyne, R. P. Surface-Enhanced Raman Spectroscopy. Annu. Rev. Anal. Chem. 2008, 1, 601-626.

(2) Moskovits, M. Surface-Enhanced Raman Spectroscopy: A Brief Retrospective. J. Raman Spectrosc. 2005, 36, 485-496.

(3) Nie, S. Probing Single Molecules and Single Nanoparticles by Surface-Enhanced Raman Scattering. Science. 1997, 275, 1102-1106.

(4) Kneipp, K.; Wang, Y.; Kneipp, H.; Perelman, L. T.; Itzkan, I. Single Molecule Detection Using SurfaceEnhanced Raman Scattering (SERS). Phys. Rev. 1997, 78, 1667-1670.

(5) Schwartzberg, A. M.; Grant, C. D.; Wolcott, A.; Talley, C. E.; Huser, T. R.; Bogomolni, R.; Zhang, J. Z. Unique Gold Nanoparticle Aggregates as a Highly Active Surface-Enhanced Raman Scattering Substrate. J. Phys. Chem. B 2004, 108, 19191-19197.

(6) Sun, L.; Song, Y.; Wang, L.; Guo, C.; Sun, Y.; Li, Z.; Liu, Z. Ethanol-Induced Formation of Silver Nanoparticle Aggregates for Highly Active SERS Substrates and Application in DNA Detection. J. Phys. Chem. C 2008, 112, 1415-1422.

(7) Yang, N.; You, T.-T.; Liang, X.; Zhang, C.-M.; Jiang, L.; Yin, P.-G. An Ultrasensitive near-Infrared Satellite SERS Sensor: DNA Self-Assembled Gold Nanorod/nanospheres Structure. RSC Adv. 2017, 7.

(8) Ahmed, S. R.; Kim, J.; Tran, V. T.; Suzuki, T.; Neethirajan, S.; Lee, J.; Park, E. Y. In Situ Self-Assembly of Gold Nanoparticles on Hydrophilic and Hydrophobic Substrates for Influenza Virus-Sensing Platform. Sci. Rep. 2017, 7, 44495.

(9) Xu, H. X.; Bjerneld, E. J.; Käll, M.; Börjesson, L. Spectroscopy of Single Hemoglobin Molecules by Surface Enhanced Raman Scattering. Phys. Rev. Lett. 1999, 83, 4357-4360.

(10) Willets, K. A.; Wilson, A. J.; Sundaresan, V.; Joshi, P. B. Super-Resolution Imaging and Plasmonics. 
(11) Yilmaz, M.; Babur, E.; Ozdemir, M.; Gieseking, R. L.; Dede, Y.; Tamer, U.; Schatz, G. C.; Facchetti, A.; Usta, H.; Demirel, G. Nanostructured Organic Semiconductor Films for Molecular Detection with Surface-Enhanced Raman Spectroscopy. Nat. Mater. 2017, 16, 918-924.

(12) Mirkin, C. A.; Letsinger, R. L.; Mucic, R. C.; Storhoff, J. J. A DNA-Based Method for Rationally Assembling Nanoparticles into Macroscopic Materials. Nature. 1996, 607-609.

(13) Aslan, K.; Luhrs, C. C.; Pérez-Luna, V. H. Controlled and Reversible Aggregation of Biotinylated Gold Nanoparticles with Streptavidin. J. Phys. Chem. B 2004, 108, 15631-15639.

(14) Faulds, K.; Littleford, R. E.; Graham, D.; Dent, G.; Smith, W. E. Comparison of Surface-Enhanced Resonance Raman Scattering from Unaggregated and Aggregated Nanoparticles. Anal. Chem. 2004, 76, 592-598.

(15) Fan, J. A.; Wu, C.; Bao, K.; Bao, J.; Bardhan, R.; Halas, N. J.; Manoharan, V. N.; Nordlander, P.; Shvets, G.; Capasso, F. Self-Assembled Plasmonic Nanoparticle Clusters. Science. 2010, 328, 1135-1138.

(16) Fang, Y.; Seong, N.-H.; Dlott, D. D. Measurement of the Distribution of Site Enhancements in Surface-Enhanced Raman Scattering. Science. 2008, 321, 388-392.

(17) Lin, L.; Peng, X.; Wang, M.; Scarabelli, L.; Mao, Z.; Liz-Marzán, L. M.; Becker, M. F.; Zheng, Y. LightDirected Reversible Assembly of Plasmonic Nanoparticles Using Plasmon-Enhanced Thermophoresis. ACS Nano 2016, 10, 9659-9668.

(18) Patra, P. P.; Chikkaraddy, R.; Thampi, S.; Tripathi, R. P. N.; Kumar, G. V. P. Large-Scale Dynamic Assembly of Metal Nanostructures in Plasmofluidic Field. Faraday Discuss. 2016, 186, 95-106.

(19) Patra, P. P.; Chikkaraddy, R.; Tripathi, R. P. N.; Dasgupta, A.; Kumar, G. V. P. Plasmofluidic SingleMolecule Surface-Enhanced Raman Scattering from Dynamic Assembly of Plasmonic Nanoparticles. Nat. Commun. 2014, 5.

(20) Yuan, Y.; Lin, Y.; Gu, B.; Panwar, N.; Tjin, S. C.; Song, J.; Qu, J.; Yong, K. T. Optical Trapping-Assisted SERS Platform for Chemical and Biosensing Applications: Design Perspectives. Coordination Chemistry Reviews. 2017, 339, 138-152.

(21) Herrmann, L.; Valev, V.; Aizpurua, J.; Baumberg, J. J. Self-Sifting of Chain Plasmons: The Complex Optics of Au Nanoparticle Clusters. Opt. Express 2013, 21, 32377-32385.

(22) Esteban, R.; Taylor, R. W.; Baumberg, J. J.; Aizpurua, J. How Chain Plasmons Govern the Optical Response in Strongly Interacting Self-Assembled Metallic Clusters of Nanoparticles. Langmuir 2012, $28,8881-8890$.

(23) Chikkaraddy, R.; de Nijs, B.; Benz, F.; Barrow, S. J.; Scherman, O. A.; Rosta, E.; Demetriadou, A.; Fox, P.; Hess, O.; Baumberg, J. J. Single-Molecule Strong Coupling at Room Temperature in Plasmonic Nanocavities. Nature 2016, 535, 127-130.

(24) Barrow, S. J.; Kasera, S.; Rowland, M. J.; Del Barrio, J.; Scherman, O. A. Cucurbituril-Based Molecular Recognition. Chemical Reviews. 2015, 7, 12320-12406.

(25) Taylor, R. W.; Lee, T. C.; Scherman, O. A.; Esteban, R.; Aizpurua, J.; Huang, F. M.; Baumberg, J. J.; Mahajan, S. Precise Subnanometer Plasmonic Junctions for SERS within Gold Nanoparticle Assemblies Using Cucurbit[n]uril "glue." ACS Nano 2011, 5, 3878-3887.

(26) Strickland, A. D.; Batt, C. A. Detection of Carbendazim by Surface-Enhanced Raman Scattering Using Cyclodextrin Inclusion Complexes on Gold Nanorods. Anal. Chem. 2009, 81, 2895-2903.

(27) Ouyang, L.; Zhu, L.; Ruan, Y.; Tang, H. Preparation of a Native $\beta$-Cyclodextrin Modified Plasmonic Hydrogel Substrate and Its Use as a Surface-Enhanced Raman Scattering Scaffold for Antibiotics Identification. J. Mater. Chem. C 2015, 3, 7575-7582.

(28) Husken, N.; Taylor, R. W.; Zigah, D.; Taveau, J. C.; Lambert, O.; Scherman, O. A.; Baumberg, J. J.; Kuhn, A. Electrokinetic Assembly of One-Dimensional Nanoparticle Chains with cucurbit[7]uril Controlled Subnanometer Junctions. Nano Lett. 2013, 13, 6016-6022.

(29) Chen, S. Y.; Mock, J. J.; Hill, R. T.; Chilkoti, A.; Smith, D. R.; Lazarides, A. A. Gold Nanoparticles on Polarizable Surfaces as Raman Scattering Antennas. ACS Nano 2010, 4, 6535-6546.

(30) de Nijs, B.; Bowman, R. W.; Herrmann, L. O.; Benz, F.; Barrow, S. J.; Mertens, J.; Sigle, D. O.; Chikkaraddy, R.; Eiden, A.; Ferrari, A.; Scherman, O. A.; Baumberg, J. J. Unfolding the Contents of 
Sub-Nm Plasmonic Gaps Using Normalising Plasmon Resonance Spectroscopy. Faraday Discuss. 2015, 178, 185-193.

(31) Salmon, A. R.; Esteban, R.; Taylor, R. W.; Hugall, J. T.; Smith, C. A.; Whyte, G.; Scherman, O. A.; Aizpurua, J.; Abell, C.; Baumberg, J. J. Monitoring Early-Stage Nanoparticle Assembly in Microdroplets by Optical Spectroscopy and SERS. Small 2016, 12, 1788-1796.

(32) Sigle, D. O.; Kasera, S.; Herrmann, L. O.; Palma, A.; De Nijs, B.; Benz, F.; Mahajan, S.; Baumberg, J. J.; Scherman, O. A. Observing Single Molecules Complexing with Cucurbit[7]uril through Nanogap Surface-Enhanced Raman Spectroscopy. J. Phys. Chem. Lett. 2016, 7, 704-710.

(33) Johnson, P. B.; Christy, R. W. Optical Constants of the Noble Metals. Phys. Rev. B 1972, 6, 43704379.

(34) Palik, E. D. Handbook of Optical Constants of Solids; 1985; 513.

(35) Taylor, R. W.; Esteban, R.; Mahajan, S.; Aizpurua, J.; Baumberg, J. J. Optimizing SERS from Gold Nanoparticle Clusters: Addressing the Near Field by an Embedded Chain Plasmon Model. J. Phys. Chem. C 2016, 120, 10512-10522.

(36) Mertens, J.; Kleemann, M. E.; Chikkaraddy, R.; Narang, P.; Baumberg, J. J. How Light Is Emitted by Plasmonic Metals. Nano Lett. 2017, 17, 2568-2574.

(37) Hugall, J. T.; Baumberg, J. J. Demonstrating Photoluminescence from Au Is Electronic Inelastic Light Scattering of a Plasmonic Metal: The Origin of SERS Backgrounds. Nano Letters. 2015, 2600-2604.

(38) Kasera, S.; Herrmann, L. O.; del Barrio, J.; Baumberg, J. J.; Scherman, O. A.; Barrio, J. del; Baumberg, J. J.; Scherman, O. A. Quantitative Multiplexing with Nano-Self-Assemblies in SERS. Sci. Rep. 2014, 4, 6785. 
For Table of Contents Use Only

\section{Mapping SERS in CB:Au Plasmonic Nano-Aggregates}

Cloudy Carnegie ${ }^{1}$, Rohit Chikkaraddy ${ }^{1}$, Felix Benz ${ }^{1}$, Bart de Nijs ${ }^{1}$, William Deacon ${ }^{1}$, Matthew Horton ${ }^{1}$, Wenting Wang ${ }^{1,2}$, Charlie Readman ${ }^{1,2}$, Steven J. Barrow ${ }^{2}$, Oren A Scherman ${ }^{2}$, Jeremy J. Baumberg ${ }^{*}, 1$

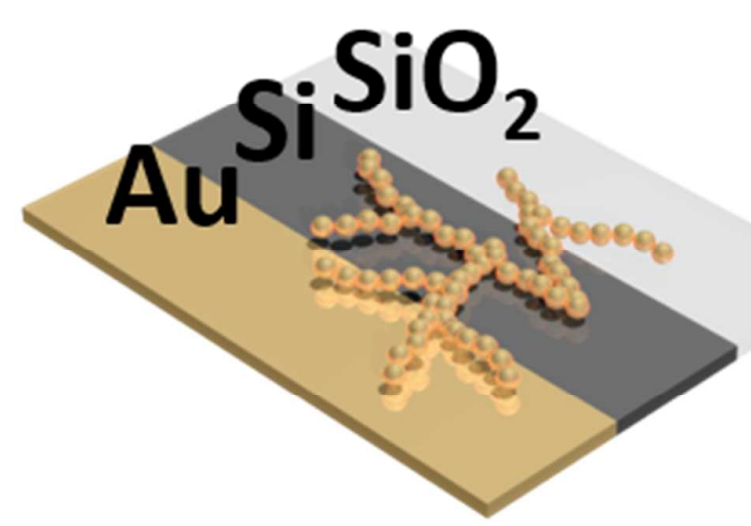

Graphic visualising a gold nanoparticle aggregate on substrates of gold, silicon and silicon dioxide.

\footnotetext{
*jjb12@cam.ac.uk
} 